\title{
Le compagnonnage cognitif : une approche pédagogique à explorer pour le développement du raisonnement clinique infirmier? - Cognitive Companionship: a potential pedagogical approach to developing clinical reasoning in nursing?
}

Marie-France Deschênes

Université de Montréal, marie-france.deschenes@umontreal.ca

Louise Boyer

University of Montreal, louise.boyer@umontreal.ca

Nicolas Fernandez

University of Montreal, nicolas.fernandez@umontreal.ca

Johanne Goudreau

University of Montreal, johanne.goudreau@umontreal.ca

Follow this and additional works at: https://qane-afi.casn.ca/journal

Part of the Educational Administration and Supervision Commons, Educational Assessment, Evaluation, and Research Commons, Educational Methods Commons, Medical Education Commons, and the Nursing Commons

\section{Recommended Citation}

Deschênes, Marie-France; Boyer, Louise; Fernandez, Nicolas; and Goudreau, Johanne (2018) "Le compagnonnage cognitif : une approche pédagogique à explorer pour le développement du raisonnement clinique infirmier? - Cognitive Companionship: a potential pedagogical approach to developing clinical reasoning in nursing?," Quality Advancement in Nursing Education - Avancées en formation infirmière: Vol. 4: Iss. 2, Article 5.

DOI: https://doi.org/10.17483/2368-6669.1156

This Article is brought to you for free and open access by Quality Advancement in Nursing Education - Avancées en formation infirmière. It has been accepted for inclusion in Quality Advancement in Nursing Education - Avancées en formation infirmière by an authorized editor of Quality Advancement in Nursing Education - Avancées en formation infirmière. 
Le compagnonnage cognitif : une approche pédagogique à explorer pour le développement du raisonnement clinique infirmier? - Cognitive Companionship: a potential pedagogical approach to developing clinical reasoning in nursing?

\section{Cover Page Footnote}

La rédaction de cet article a été rendu possible par des bourses doctorales du Conseil de recherches en sciences humaines du Canada (CRSH), du Centre d'innovation en formation infirmière (CIFI) de I'Université de Montréal ainsi que de l'Équipe FUTUR, infrastructure de recherche subventionnée par le FRQ-SC. This article's publication was made possible thanks to doctoral awards from the Social Sciences and Humanities Research Council of Canada (SSHRC), Université de Montréal's CIFI (Centre d'innovation en formation infirmière) and the FUTUR team, a research infrastructure subsidized by the FRQSC. 


\section{Introduction}

Un des défis actuels des institutions d'enseignement supérieur est de concevoir et de planifier l'articulation des activités éducatives pour favoriser le développement optimal des compétences visées par le programme, dont le raisonnement clinique infirmier (RCI). Le RCI regroupe les " processus cognitifs complexes d'utilisation de la cognition, de la métacognition et des savoirs disciplinaires pour rassembler et analyser les données, évaluer la signification des données recueillies afin de décider d'interventions cliniques »[traduction libre] (Simmons, 2010, p. 1155). Or, le découpage entre la théorie et la pratique du RCI demeure un enjeu pédagogique qui persiste dans les programmes de formation. Les connaissances acquises pendant la formation ne sont pas d'emblée mobilisées pendant l'action clinique (Benner, Sutphen, Leonard, et Day, 2010; Levett-Jones et al., 2010). Précisément, les lacunes liées au RCI et notées chez les novices concernent, entre autres, l'identification des données pertinentes à la compréhension d'une situation de soin (Alfaro-LeFevre, 2013) ainsi que l'anticipation d'une détérioration clinique (Levett-Jones et al., 2010). Une faible génération d'hypothèses cliniques est aussi observée (Boyer, Tardif, et Lefebvre, 2015; Goudreau, Boyer, et Létourneau, 2014) alors qu'elle est une composante indispensable au RCI. Puisqu'il est partie prenante de la qualité des soins dispensés à la population (Alfaro-LeFevre, 2013; Thompson, Aitken, Doran, et Dowding, 2013), le développement du RCI chez les étudiantes constitue une des priorités des institutions d'enseignement supérieur. Dès lors, il s'avère essentiel de repenser la qualité de l'accompagnement pédagogique par une cohérence plus affinée entre la formation et la pratique d'un RCI contextualisé en situation authentique de la pratique professionnelle.

À cet égard, le compagnonnage cognitif semble être une approche prometteuse pour le développement de cette compétence. Le compagnonnage cognitif se traduit par l'établissement d'une relation pédagogique et dialogique entre l'experte formatrice ${ }^{1}$ et l'étudiante où les stratégies cognitives et métacognitives déployées à la résolution d'un problème en situation authentique sont exploitées et socialement partagées (Bédard, Frenay, Turgeon et Paquay, 2000; Collins, Brown, et Holum, 1991; Collins, Brown, et Newman, 1989; Frenay et Bédard, 2004; Vanpee, Frenay, Godin, et Bédard, 2010). Ancré dans une perspective sociocognitiviste, le compagnonnage cognitif se distingue du compagnonnage simple par ses composantes relationnelles et dialogiques. Dans le compagnonnage simple, la relation et les interactions entre les étudiantes et les expertes sont de moindre importance ou peu exploitées ; les étudiantes apprennent par observation ou par mimétisme (Poteaux et Pelaccia, 2016). L'étalage des processus cognitifs et métacognitifs utilisés par les expertes dans des situations authentiques de la pratique est ainsi peu exposé ou exploité (Benner et al., 2010; Dennen et Burner, 2008; Vanpee et al., 2010) : les étudiantes y accèdent rarement ou sporadiquement. En d'autres mots, le savoir tacite des expertes demeure trop souvent silencieux et les étudiantes n'obtiennent pas d'explication, de justification ou d'articulation intentionnelle des hypothèses et des interventions cliniques avancées par les expertes dans des situations authentiques de la pratique professionnelle (Benner et al., 2010). Inconsciemment mais fréquemment engagées dans un compagnonnage simple, les expertes en sciences infirmières se butent à des difficultés récurrentes en matière de RCI chez les étudiantes.

\footnotetext{
${ }^{1}$ Les termes experte formatrice englobent également le genre masculin. Ils désignent toute personne qui intervient dans la formation des étudiantes, notamment les professeures, les tutrices, les préceptrices, les enseignantes, les expertes cliniques, etc. Afin de favoriser la lecture, le terme experte sera subséquemment utilisé dans le texte.
} 
C'est dans cette perspective que cet article, rédigé sous la forme d'un essai, propose l'étude du compagnonnage cognitif comme approche pédagogique pour soutenir le développement du RCI, et ce, par l'utilisation de diverses stratégies pédagogiques favorables au développement de la compétence. La première section de l'article aborde les fondements épistémologiques et les théories de l'apprentissage qui sous-tendent le développement du RCI. Ensuite, sera mise en exergue la contribution potentielle du compagnonnage cognitif comme une approche pédagogique à explorer pour le développement de la compétence. Des stratégies pédagogiques sont énumérées et exemplifiées par les auteurs afin d'illustrer l'utilisation du compagnonnage cognitif. En conclusion, une discussion considère les défis et les retombées de cette approche.

\section{Repères épistémologiques et théoriques au développement du RCI}

L'épistémologie est l'étude des modes de raisonnement utilisés pour le développement des connaissances (Lecourt, 2010). Les repères épistémologiques et théoriques présentés cidessous permettent de saisir les différentes conceptions de l'apprentissage liés au développement du RCI. Le compagnonnage cognitif prend appui sur ces repères notamment la médiation sociale et l'effort dialogique réflexif nécessaires au développement du RCI.

\section{Les repères épistémologiques}

Le socioconstructivisme et le cognitivisme seront mis en évidence dans cette section afin de mieux saisir l'apport de la cognition située et de l'utilisation du compagnonnage cognitif comme une approche pédagogique à explorer pour le développement du RCI.

Le socioconstructivisme, paradigme avancé par Vygotsky (Vygotsky, 1933/2013), suggère une construction des connaissances, et ce, principalement par l'interaction sociale du sujet avec son environnement. L'acquisition des connaissances fait partie d'une démarche collective d'élaboration et de négociation de sens, une conception socio-historico-culturelle des objets de la connaissance. Ce paradigme met de l'avant les outils culturels de communication (ex. : le langage) et la fonction d'étalage des connaissances. En d'autres mots, il est nécessaire d'établir un dialogue et une médiation sociale pour acquérir des connaissances et pour se développer. À ce titre, les orientations pédagogiques de Vygostky insistent sur la culture, la dimension historique et les interactions sociales comme des éléments constitutifs et imbriqués au développement de la pensée (Legendre, 2005). Cette notion est fondamentale dans le processus de développement des savoirs infirmiers. Engagée dans un devenir professionnel, la démarche pédagogique revêt ainsi un caractère social qui teinte la signification cognitive associée aux savoirs infirmiers en processus de développement.

Le cognitivisme est une perspective où l'on étudie les processus mentaux liés à l'acquisition, la manipulation, la mémorisation et la production des connaissances (Legendre, 2004). Cet aspect computationnel est à l'image d'une carte cognitive qui guide la captation, le traitement des informations et leurs utilisations subséquentes. Les connaissances acquises, les schèmes de représentations ou les scripts mentaux agissent, en quelque sorte, comme des grilles de lecture pour l'analyse d'une situation. Le sujet aborde pertinemment une situation en se référant à son bagage expérientiel mémorisé sous forme de modèles ou de schémas de représentations mentales (Schank et Abelson, 1977). Cette propension à user de représentations mentales permet au sujet de reconnaître des situations vécues, d'interpréter, de prédire des évènements et d'agir intuitivement (Frith, 2007). La contribution des connaissances est ainsi intimement liée au RCI (Bordage, 1994; Nendaz, Charlin, Leblanc, et Bordage, 2005). 
Lorsqu'elles abordent des situations professionnelles, les expertes utilisent des structures cognitives conceptualisées sous la forme de réseaux de connaissances élaborées, structurées et significativement liées à la pratique. Ces réseaux de connaissances ou scripts mentaux sont dès lors définis comme des schémas de représentation mentale qui permettent de stéréotyper en unités de sens différentes informations liées à une situation (Schank et Abelson, 1977).

\section{Les repères théoriques au développement du RCI}

Le RCI se caractérise par les processus métacognitifs et cognitifs récursifs engagés par l'infirmière. Un répertoire de connaissances solides et une pratique professionnelle réflexive favorisent le développement du RCI (Goudreau et al., 2014; Simmons, 2010). À cet égard, une compréhension des repères théoriques liant le développement des connaissances à l'expérience professionnelle permet de saisir la contribution potentielle du compagnonnage cognitif au développement du RCI.

Appuyé sur une pratique professionnelle, le développement de l'expertise en matière de RCI repose sur un répertoire de savoirs élaborés et finement organisés au cours des années (Boyer et al., 2015; Goudreau et al., 2014; Simmons, 2010). Les infirmières expertes sont en quelque sorte porteuses d'un savoir tacite : elles possèdent un large registre de situations professionnelles qui leur permet d'orienter et de réguler leur pratique, et ce, par l'utilisation de diverses stratégies cognitives (Fonteyn et Ritter, 2008). Elles pratiquent de manière proactive, recueillant un large éventail de repères pour reconnaître avec exactitude les indices cliniques, comprendre une situation de soins, intervenir adéquatement et prévenir les éventuelles conséquences ou complications chez le patient (Hoffman, Aitken, et Duffield, 2009; Sarsfield, 2014; Simmons, Lanuza, Fonteyn, Hicks, et Holm, 2003). En d'autres mots, les expertes ont acquis de riches réseaux de savoirs professionnels, appelés scripts mentaux pour aborder une situation de soins et ainsi utiliser un RCI solide et situé (Deschênes et Goudreau, 2017). Un script désigne les réseaux associatifs, le tissage des liens entre les savoirs qui guident la perception, la sélection, l'interprétation et la mémorisation d'informations (Charlin, Tardif, et Boshuizen, 2000; Schmidt, Norman, et Boshuizen, 1990). Alors que les scripts sont élaborés et compilés chez l'experte, ils sont plus dispersés ou réduits chez l'étudiante ou la novice (Bordage, 1994; Nendaz et al., 2005). Par ailleurs, les scripts sont développés dès que l'étudiante commence à être confrontée à des situations authentiques de la pratique professionnelle : ils s'élaborent et se raffinent par le développement continu de l'expertise professionnelle (Charlin et al., 2000; Schmidt et al., 1990). Le développement professionnel, dans de telles circonstances, dépend entre autres, de mécanismes d'autorégulation cognitive comme la pratique réflexive (Argyris et Schön, 1992/1974), une pratique métacognitive qui permet de repenser l'action clinique et de se penser à travers cette action. Ainsi, le développement de l'expertise ne se produit pas de façon passive, fortuitement et sans compagnonnage (Ericsson, 2008). Bien qu'elle s'inscrive dans l'exercice processuel du RCI, la pratique réflexive n'est pas un exercice automatisé ni exclusivement individualisé. Dès lors, il apparaît essentiel de solliciter et de soutenir des activités éducatives actives qui misent sur une pratique réflexive délibérée en situation authentique (Lavoie, Boyer, Pepin, Goudreau, et Fima, 2017) et sur un dialogue cognitif socialement partagé entre étudiantes et expertes, afin de favoriser le développement professionnel continu du RCI.

Maintenant, comment les repères épistémologiques et théoriques peuvent-ils aider à mieux conceptualiser les stratégies éducatives à mettre en place pour le développement de la 
compétence ? Comment transposer ces repères à la qualité de l'accompagnement pédagogique en sciences infirmières?

En pédagogie, penser comme un sociocognitiviste signifie une propension à utiliser une variété de situations d'apprentissage et à multiplier ces occasions pédagogiques (Tardif, 2016). Dans cette perspective, il n'y a pas de réelle élaboration des connaissances sans qu'une activité cognitive soit sollicitée (Legendre, 2004). On maximise les occasions pédagogiques qui mettent en branle les capacités cognitives des étudiantes (Tardif, 2016), comme le tissage de liens entre les connaissances et l'organisation optimale de celles-ci. Il est souhaité que l'étudiante transforme les informations en connaissances viables, afin que celles-ci soient progressivement intégrées à des schèmes cognitifs (Tardif, 1992, 1999). L'accessibilité aux connaissances dans la mémoire à long terme et la rétention de celles-ci sont liées au principe d'itération (Lasnier, 2000), c'est à dire à la fréquence des rappels pédagogiques générés par des stratégies de consolidation et de réinvestissement des connaissances (Bisonnette et Richard, 2005; Tardif, 2016). Le défi pédagogique est de mettre en branle le début d'une matrice architecturale de savoirs professionnels en processus de développement. L'experte considère la nécessité des interactions sociales à la construction et à la négociation de sens qui teintent la construction des connaissances (Legendre, 2004; Legendre, 2005; Tardif, 2016). L'élaboration des connaissances engage un déséquilibre sociocognitif chez l'étudiante alors qu'elle est en relation avec son environnement. Dans le cadre de l'élaboration des connaissances visant le développement de l'expertise professionnelle, cette négociation de sens est particulièrement éclairante. Elle s'inscrit dans une zone proximale de développement, notion vygotskienne de déséquilibre sociocognitif visant le développement de l'être en devenir (Vygotsky, 1978). Alors qu'elle ne parvient pas à effectuer une tâche seule, l'étudiante y arrive avec l'aide d'une experte ou d'une communauté d'apprentissage. Il s'agit de la création d'un espace pédagogique visant le développement potentiel de l'étudiante tout en misant sur ses capacités actuelles.

À cet égard, la perspective de la cognition située suggère des pistes pédagogiques qui permettent d'éclairer et d'opérationnaliser ces concepts. Elle est une perspective privilégiée comme mode d'accompagnement pédagogique alliant pertinemment les activités d'apprentissage/enseignement afin de favoriser le développement du RCI (Benner et al., 2010; Field, 2004; Gieselman, Stark, et Farruggia, 2000), notamment en formation initiale (Kuiper, 2013; Sadhuwong, Koraneekij, et Natakuatoong, 2016), lors de mentorat (Forneris et PedenMcAlpine, 2009; Nickle, 2006), en contexte de simulation clinique ou par l'utilisation de soutiens numériques à l'apprentissage (Sadhuwong et al., 2016; Woolley et Jarvis, 2007; Wyrostok, Hoffart, Kelly, et Ryba, 2014). La perspective de la cognition située (Brown, Collins, et Duguid, 1989) repose sur deux principes essentiels. Dans un premier temps, le traitement des informations est indissociablement lié au contexte dans lequel elles prennent place. Ceci réfère à l'importance de solliciter des situations d'apprentissage en contexte authentique. Mais puisque les expériences d'apprentissage ne sont pas forcément formatrices (Mandeville, 2009), l'accompagnement pédagogique concourt à l'établissement d'une relation pédagogique optimale, élément déterminant à la réutilisation subséquente des connaissances chez l'étudiante (Frenay et Bédard, 2004). La notion de la cognition située suggère ainsi d'éviter des situations éducatives épurées des conditions de la réalité professionnelle (Collins et al., 1989; Vanpee et al., 2010). Au contraire, il faut miser sur des situations authentiques et signifiantes pour favoriser l'ancrage des connaissances dans la mémoire à long terme, mais aussi pour susciter la motivation chez l'étudiante. La cognition située engage également un soutien cognitif adapté et progressif aux processus et aux étapes de développement de la compétence du RCI chez l'étudiante. 
L'acquisition des connaissances repose sur un enseignement explicité où se conjuguent la supervision et le questionnement ainsi que la rétroaction constructive (Bédard et al., 2000; Frenay et Bédard, 2004; Vanpee et al., 2010), comme l'illustre le compagnonnage cognitif.

\section{Le compagnonnage cognitif}

Ancré dans une perspective sociocognitiviste, le compagnonnage cognitif est une approche pédagogique qui vise la résolution d'un problème en situation authentique ainsi que l'établissement d'une relation pédagogique entre l'experte et l'étudiante où les stratégies cognitives et métacognitives déployées sont exploitées et socialement partagées (Bédard et al., 2000; Collins et al., 1991; Collins et al., 1989; Frenay et Bédard, 2004; Vanpee et al., 2010). La finalité de ce dialogue cognitif vise le développement d'une pensée progressivement autonome et critique chez l'étudiante (Bédard et al., 2000; Dennen et Burner, 2008; Frenay et Bédard, 2004) ainsi que l'établissement d'une communauté d'apprentissage. La communauté d'apprentissage se conceptualise par la création d'un environnement dans lequel les participantes communiquent, collaborent et s'engagent activement au développement mutuel des compétences. Une telle communauté conduit à un sentiment d'appartenance, caractérisé par l'investissement personnel et par l'interdépendance éducative entre les différents participantes (Collins et al., 1991; Wenger, 1998). Pour atteindre ces finalités, certaines conditions sont associées à l'approche pédagogique du compagnonnage cognitif, elles mettent en lumière la responsabilité partagée entre l'étudiante et l'experte quant au développement des connaissances et à leurs utilisations judicieuses en contexte situé (Figure 1).

Figure 1

Les principes du compagnonnage cognitif au développement $d u R C I$

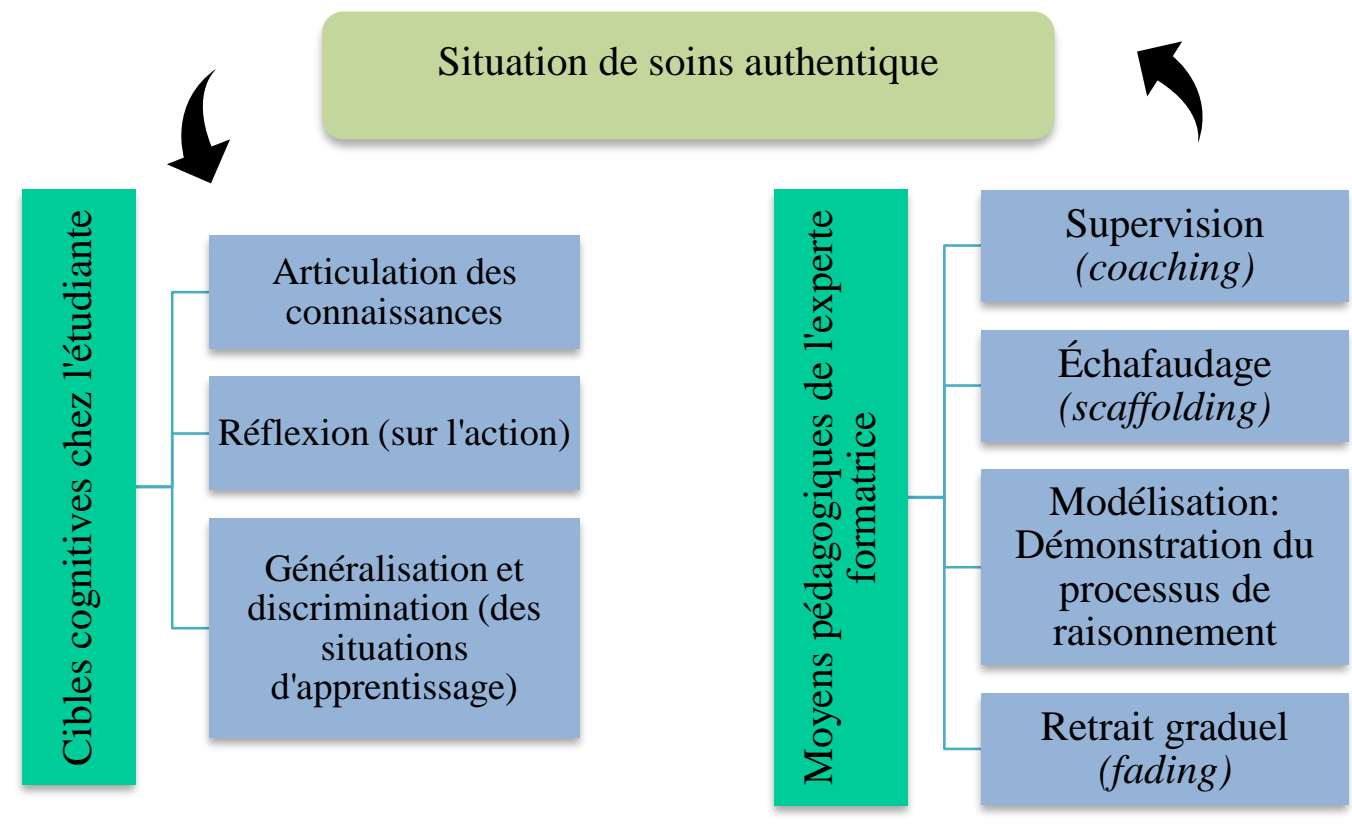

Communauté d'apprentissage

Note : Adaptation de Frenay et Bédard p.251 (2004) 
Dans tout compagnonnage cognitif, l'experte agit comme une médiatrice : elle observe, guide, donne une rétroaction, explore et questionne la démarche de l'étudiante (Collins et al., 1991; Frenay et Bédard, 2004; Vanpee et al., 2010). Elle agit de façon ponctuelle avec celle-ci en l'aidant à réfléchir et à questionner ses stratégies cognitives utilisées en situation clinique authentique. Elle engage un dialogue cognitif tout en précisant à voix haute ses propres stratégies de résolution de problème. Par exemple, l'experte explicite ses prises de décisions cliniques en situation authentique par l'énonciation des nuances et des subtilités fines qui guident son processus de résolution de problème. L'approche du compagnonnage cognitif permet ainsi de saisir les contingences d'une situation clinique authentique qui guident le choix décisionnel des expertes. Elle permet de capter leurs « savoirs-vivants », ces savoirs spécialisés et construits par la pratique quotidienne, mais qui demeurent difficilement traduits ou absents des ouvrages de référence (Charlin et Fernandez, 2016). Graduellement, l'experte laisse de la liberté à l'étudiante afin que celle-ci agisse de façon autonome à l'exercice du RCI.

Le compagnonnage cognitif propose des moyens pédagogiques pour accompagner pertinemment l'étudiante. Par exemple, la supervision (coaching) fait référence à un accompagnement serré, une supervision directe de l'étudiante tout en lui fournissant une rétroaction à la suite d'une démarche clinique ou intellectuelle complexe. La supervision est un des principes centraux du compagnonnage cognitif. Sans émettre toutes les solutions à l'étudiante, l'experte suggère des pistes de réflexion ou soulève des commentaires clés pour accompagner l'étudiante dans sa démarche (Vanpee et al., 2010). De son côté, l'échafaudage (scaffolding) suggère un accompagnement certes, mais adapté aux apprentissages de l'étudiante. Alors que la supervision est nettement plus engagée voire intrusive au départ, elle devient plus ciblée et ponctuelle par la suite. L'experte fournit des indices et agit comme facilitatrice pour optimiser graduellement la prise en charge par l'étudiante. Le soutien de l'experte varie selon le degré de développement de la compétence par l'étudiante. La modélisation (modeling) propose une démonstration étayée des processus cognitifs engagés chez l'experte à la réalisation d'une démarche complexe. Il est d'ailleurs bénéfique que l'étudiante bénéficie de plusieurs observations variées où les expertes explicitent volontairement leurs processus de pensée (Rieder, Poletti, et Audétat, 2015). Enfin, le retrait graduel (fading) suggère l'autonomisation progressive de l'étudiante. L'apport de l'experte diminue graduellement au fur et à mesure que l'étudiante met en œuvre les cibles cognitives optimales au développement du RCI et qu'elle devient de plus en plus autonome dans sa démarche. Cette responsabilité réciproque entre l'experte et l'étudiante se veut garante du développement attendu d'un RCI nécessaire à l'exercice de la profession.

Dans cette approche, l'étudiante est invitée à articuler ses connaissances, à réfléchir ainsi qu'à discriminer et généraliser les connaissances liées à des situations authentiques d'apprentissage. L'activité de réflexion pendant ou après l'activité éducative propulse l'étudiante dans une conscientisation de ses connaissances et de ses stratégies cognitives utilisées pour la résolution de problème. La pratique réflexive occupe une place essentielle dans cette approche pédagogique. L'articulation des connaissances et le tissage de liens entre celles-ci soutiennent des apprentissages en profondeur (Jouquan, Romanus, Vierset, Jafferelot, et Parent, 2013). Les activités de discrimination et de généralisation permettent l'amorce d'un encodage des savoirs dans la mémoire à long terme. Certains schémas de représentations mentales sont graduellement mis en branle. Ils peuvent être, par la suite, réutilisés dans d'autres situations authentiques similaires (Custers, 2015; Lubarsky, Dory, Audétat, Custers, et Charlin, 2015). D'autres fois, des éléments-clés distinguent des situations authentiques dites atypiques. Ils peuvent aussi marquer 
un encodage spécifique des représentations mentales en élaboration chez l'étudiante (Custers, 2015; Lubarsky et al., 2015). Ainsi, il est suggéré d'accroitre graduellement la complexité et la diversité des situations d'apprentissage et de présenter une vision globale et intégrative d'une démarche clinique à l'étudiante avant de la situer face à une démarche spécifique à déployer (Collins et al., 1991; Collins et al., 1989; Vanpee et al., 2010). Des apprentissages en surface et le morcellement de ceux-ci sont ainsi évités, car l'étudiante sera en mesure de lier sa démarche à un cadre plus global et ce cadre doit être significativement associé à une situation authentique de la pratique professionnelle.

Maintenant, comment rendre opérationnelle une telle intention pédagogique en formation en sciences infirmières? Par quelles stratégies éducatives peut-on s'inscrire dans une approche de compagnonnage cognitif?

\section{Exemples d'application du compagnonnage cognitif}

Quelques stratégies éducatives peuvent s'appuyer sur l'approche du compagnonnage cognitif. Citons, par exemple, les exercices d'explication ou de questionnement en milieu clinique, les séances d'apprentissage par problèmes, les séances de débreffage lors d'immersions en simulation clinique, la schématisation interactive, etc. Récemment, des travaux de recherche sur la formation par concordance (Charlin et Fernandez, 2016; Fernandez et al., 2016; Foucault, Dubé, Fernandez, Gagnon, et Charlin, 2015) permettent aussi d'envisager des pistes de réflexion intéressantes, notamment sur ce mode d'accompagnement pédagogique en situation authentique de pratique complexe. Une formation par concordance est basée sur l'utilisation formative d'un test de concordance de scripts (TCS) et elle comprend plusieurs scénarios cliniques rédigés à partir de problèmes mal définis (Charlin et Fernandez, 2016). Ces scénarios visent à évaluer le raisonnement clinique de l'étudiante dans un contexte d'ambigüité et d'incertitude, illustrant des circonstances professionnelles authentiques et de comparer son raisonnement à ceux d'un panel d'expertes ayant répondu préalablement aux scénarios présentés (Charlin et Fernandez, 2016; Fernandez et al., 2016; Foucault et al., 2015). La structure de présentation de l'outil de formation par concordance est rédigée à l'image du processus cognitif hypothético-déductif du RCI (Charlin, Gagnon, Sibert, et Van der Vleuten, 2002; Charlin et Van der Vleuten, 2004) où les scripts sont activités et mobilisés à la résolution d'un problème. Les activités de formation par concordance visent à étayer les savoirs des expertes en passant par une explication détaillée des réflexions de ces dernières lorsqu'elles complètent les scénarios cliniques de la formation. L'étudiante est en mesure de valider si les décisions prises dans les processus de raisonnement des expertes concordent avec les siennes, d'où la notion concordance de scripts. D'autres outils d'évaluation formative auxquels s'incorpore une rétroaction par des expertes peuvent s'appuyer sur l'approche du compagnonnage cognitif en formation infirmière. Les avancées technologiques actuelles permettent l'élaboration d'une myriade de stratégies éducatives pour aborder un compagnonnage cognitif dit numérique, notamment les cyber apprentissages, les forums d'échanges, les webinaires, les jeux sérieux (serious games) ou les jeux interactifs, etc. L'emploi d'environnements numériques à l'apprentissage rend facilement accessible le recours d'expertes du domaine en plus de joindre un nombre important d'étudiantes (Fernandez et al., 2016).

En conclusion, le tableau 1 expose une variété d'exemples qui peuvent être employés par les expertes dans l'approche du compagnonnage cognitif, et ce, par les différents moyens pédagogiques énumérés précédemment. Basés sur une vaste expérience de pratique pédagogique et de supervision clinique, ces exemples témoignent de moyens pédagogiques jugés pertinents par les auteurs. 
Tableau 1

Moyens pédagogiques du compagnonnage cognitif

\section{Buts \\ Exemples}

Favoriser les situations de supervision directe ou de mentorat périphérique
Expliquer et résumer les processus de pensée par la verbalisation à voix haute (experte et étudiante) lors de discussion de cas (réels ou fictifs), d'exercices de schématisation en groupe, de synthèses des apprentissages, de rapports interservices en milieux cliniques, etc.

Poser des questions à l'étudiante avant, pendant et après une démarche clinique ou intellectuelle complexe

- Articulation des connaissances et réflexion:Que notestu dans cette situation? Que se passe-t-il ? À quoi penses-tu ? Qu'est-ce qui t'apparaît prioritaire ? Quelles sont tes prédictions quant à l'évolution de cette situation? Sur quels éléments bases-tu ton hypothèse ou ta prise de décision? Es-tu en mesure de résumer la situation en 2-3 phrases?

- Généralisation et discrimination : As-tu déjà vécu des situations similaires? Quelles sont les différences ou similitudes remarquées? Si je retire ou si j'ajoute ce nouvel élément dans le contexte, comment cela nuance ton raisonnement? Quels sont les éléments qui confirment ou qui infirment telle ou telle hypothèse? Que manque-t-il comme information pour confirmer l'hypothèse? Y a-t-il d'autres alternatives?

Observer l'étudiante pendant qu'elle accomplit une démarche et lui offrir une rétroaction constructive et liée au contexte d'apprentissage

Animer des séances de breffage et de débreffage per et post simulation en posant des questions et en encourageant la verbalisant à voix haute chez les étudiantes. Permettre la réflexion partagée entre les étudiantes. Expliquer les zones d'ambiguïté et d'incertitude relativement à la simulation vécue. Faire émerger ses propres questionnements relativement à la situation simulée

Permettre les forums d'échange et de collaboration sur le web et favoriser tout tutorat entre étudiantes de divers niveaux de formation 
Soutenir les étudiantes à la réalisation d'une démarche complexe de la pratique clinique

Accompagner l'étudiante à l'accomplissement d'une tâche complexe en lui donnant quelques indices

Agir comme facilitatrice. Par exemple, amorcer une démarche clinique et permettre à l'étudiante de la finaliser tout en bénéficiant du soutien de l'experte

Offrir judicieusement quelques indices à la résolution de problème

Réaliser une démarche complexe devant l'étudiante et verbaliser intentionnellement à voix haute ses perceptions et ses processus de pensée suggérant des hypothèses d'explications ou d'interventions infirmières

Poursuivre le questionnement auprès de l'étudiante

Favoriser le traitement sémantique des données par l'utilisation de la pensée à voix haute (étudiante et experte). Par exemple, demander à l'étudiante de résumer une situation clinique réelle ou fictive en quelques concepts clés

Rendre explicites les processus de raisonnement des expertes du domaine
Faire une démarche complexe devant les étudiantes et décrire à voix haute les subtilités et les nuances qui guident les choix cliniques

Permettre l'observation et le dialogue avec des expertes en milieu clinique, par des vidéoconférences, des simulations de cas, des webinaires, etc. ainsi qu'une période de discussion suivant ces activités

Utiliser une évaluation formative (ex.: formation par concordance) pour exposer les réflexions des expertes à l'aide d'une plateforme web ou par des groupes de discussion

Verbaliser à voix haute les processus de pensée lors de procédures cliniques, d'apprentissage par problème, de discussion de cas cliniques, de webinaires, etc. Par exemple, résumer une situation clinique sous forme d'un rapport interservices et basé sur une pratique exemplaire (quels sont les éléments-clés énumérés et en quoi cela permet à l'infirmière de saisir rapidement l'enjeu ou la gravité clinique?)

Exploiter et exposer votre résumé d'une situation réelle ou fictive par quelques concepts clés afin que l'étudiante puisse percevoir votre traitement sémantique opéré (votre mise en mots de la situation) 


\begin{tabular}{|c|c|}
\hline \multirow{3}{*}{$\begin{array}{l}\text { Favoriser le } \\
\text { développement de } \\
\text { l'autonomie de } \\
\text { l'étudiante }\end{array}$} & $\begin{array}{l}\text { Se retirer graduellement du processus d'apprentissage de } \\
\text { l'étudiante sans négliger l'apport d'une rétroaction } \\
\text { constructive }\end{array}$ \\
\hline & $\begin{array}{l}\text { Se rendre disponible à l'étudiante tout en lui accordant une } \\
\text { certaine liberté }\end{array}$ \\
\hline & $\begin{array}{l}\text { Accompagner l'étudiante à l'édification progressive de sa } \\
\text { professionnalisation dans une culture de formation } \\
\text { continue et d'engagement à une communauté } \\
\text { d'apprentissage dans sa profession }\end{array}$ \\
\hline
\end{tabular}

\section{Discussion}

\section{Les défis et les retombées de l'approche du compagnonnage cognitif}

Cette section mettra en relief les défis que représente l'approche du compagnonnage cognitif ainsi que des suggestions pour atténuer ceux-ci. Enfin, quelques retombées potentielles du compagnonnage cognitif seront considérées pour la formation, la profession et la recherche en sciences infirmières.

\section{Les défis du compagnonnage cognitif}

Puisqu'elle engage l'extériorisation des processus de pensée, l'utilisation de l'approche du compagnonnage cognitif prédispose à des zones de vulnérabilité où l'humilité sera nécessaire autant chez les étudiantes que chez les expertes. Face à cet aspect, nous soulignons l'importance du climat de confiance (Brown et al., 1989; Collins et al., 1989; Rieder et al., 2015), de l'engagement mutuel et du partage des attentes mutuelles face à l'utilisation de l'approche du compagnonnage cognitif. Il est aussi suggéré d'accueillir avec bienveillance les représentations mentales des étudiantes, afin de potentialiser les mesures de remédiation, si nécessaire. En outre, l'attribution du modèle de rôle aux expertes demeure un autre défi de taille face à l'utilisation de cette approche pédagogique (Rieder et al., 2015). Pour plusieurs expertes, les éléments-clés qui nuancent certaines prises de décision clinique demeurent difficiles à déconstruire à voix haute, notamment à cause de l'autonomie développée au cours des années de pratique (Palmeri, 1997). De plus, les explications verbales ne reflètent pas toujours avec exactitude le processus de pensée : l'activation des scripts mentaux demeure un processus automatique et relativement inconscient (Lubarsky et al., 2015). À cet égard, il est proposé aux expertes de faire délibérément un effort d'explicitation face à une démarche complexe et de partager à voix haute les questionnements et les décisions projetées en lien avec les éléments clés décelés dans toute situation authentique de la pratique professionnelle. Les étudiantes se voient rassurées quant à la possibilité de développer les compétences professionnelles lorsqu'elles prennent conscience que les expertes usent aussi de plus d'une étape itérative de questionnement et de remise en question pendant une démarche complexe (Collins et al., 1989). Enfin, cet effort d'articulation des connaissances et la mise en relation de celles-ci permettent d'engager un certain dialogue entre étudiantes et expertes ainsi qu'une modélisation potentielle du mode de raisonnement. 


\section{Les retombées de l'approche du compagnonnage cognitif}

Le compagnonnage cognitif tend à rendre visibles les savoirs tacites des expertes à la résolution de problèmes authentiques de la pratique (Collins et al., 1991; Collins et al., 1989; Frenay et Bédard, 2004; Lyons, McLaughlin, Khanova, et Roth, 2016; Vanpee et al., 2010). Par cette démarche, il ne s'agit pas de proposer un mimétisme des activités cognitives engagées par les expertes, mais d'offrir un rapport dialogique aux savoirs infirmiers en processus d'élaboration : un étalage partagé des représentations mentales liées à une situation authentique de la pratique (Deschênes et Goudreau, 2017). Cette confrontation pédagogique suggère une réorganisation des connaissances et des stratégies cognitives de l'étudiante. Au mieux, elle la confirme dans ses hypothèses ou ses prises de décision dans la résolution de diverses situations cliniques (Poteaux et Pelaccia, 2016). Cette démarche comparative permet ainsi d'accroître les capacités métacognitives chez les étudiantes (Frenay et Bédard, 2004; Vanpee et al., 2010). Ces considérations rejoignent ce que Vygostky décrit comme la zone de développement proximal. Il s'agit de cette zone de rapport dynamique et dialogique entre les représentations du sujet et la négociation de sens des savoirs socialement partagés et faisant l'objet d'une transmission éducative (Vygotsky, 1933/2013; Vygotsky, 1978). Dès lors, la rétroaction occupe une place essentielle afin de situer les étudiantes dans leur processus de développement professionnel (Vanpee et al., 2010). Sans rétroaction, des conceptions erronées, une inaptitude à déceler une erreur ou de mauvaises habitudes peuvent être développées chez les étudiantes (O'Brien, Marks, et Charlin, 2003). Enfin, une identification rapide des apprentissages du raisonnement clinique effectué ou non chez les étudiantes est nécessaire afin de les guider adéquatement et d'éviter des impasses pédagogiques où une cristallisation des difficultés s'est forgée avec le temps (Audétat, Laurin, et Sanche, 2011; Audetat et al., 2013).

Le compagnonnage cognitif se veut quelques fois pluriel, et ce, par le recours à divers modèles de rôle dans l'accompagnement au développement du RCI. La création d'une communauté d'apprentissage ou d'une communauté de pratique (Lave et Wenger, 1991; Wenger, 1998) à l'exercice d'une profession est un effet collatéral généré par différentes stratégies éducatives qui misent sur le compagnonnage cognitif. Nul doute que l'intention pédagogique sous-jacente concourt à ce partage des savoirs spécialisés et développés au cours des années par les expertes, une modélisation cognitive basée sur une réelle expertise dans la profession. De plus, elle permet une participation légitime périphérique (Lave, 1997; Lave et Wenger, 1991): une reconnaissance et une contribution des expertes du domaine à la construction des savoirs de la discipline pour favoriser le développement optimal du raisonnement chez les étudiantes, et ce, avant leur accès à la profession. Enfin, elle assure une transition harmonieuse entre les savoirs et l'application subséquente de ceux-ci à la pratique clinique (Fernandez et al., 2016).

Différentes recherches et interventions pédagogiques dans les milieux cliniques pourraient être conduites afin d'établir un lien cohérent entre l'approche du compagnonnage cognitif, les stratégies éducatives utilisées et le développement du RCI. Par exemple, comment mieux ancrer le compagnonnage cognitif dans une culture organisationnelle afin d'améliorer la qualité du préceptorat dans les milieux académiques et cliniques ? Comment les environnements numériques à l'apprentissage s'inscrivent-ils dans une approche de compagnonnage cognitif ? Comment arrimer un modèle cognitif d'apprentissage du RCI, comme le propose Goudreau et al. (2014) aux stratégies éducatives mises en œuvre dans les programmes de formation et qui seraient liées aux principes du compagnonnage cognitif ? 


\section{Conclusion}

Au-delà du compagnonnage simple, le compagnonnage cognitif implique le dialogue et l'extériorisation des processus cognitifs contributifs au développement du RCI. Il permet de saisir les contingences d'une situation clinique authentique qui guident le choix décisionnel des expertes et de capter en quelque sorte leurs "savoirs-vivants », ces savoirs spécialisés et construits par la pratique quotidienne, mais qui demeurent difficilement traduits ou absents des ouvrages de référence (Charlin et Fernandez, 2016). Différentes stratégies qui s'inscrivent dans une perspective de compagnonnage cognitif pourraient permettre une transition optimisée entre les connaissances et l'univers de la pratique clinique. Il est d'ailleurs souhaité que les stratégies éducatives mises en place aspirent à la professionnalisation et qu'elles suggèrent la possibilité aux étudiantes de penser comme une infirmière. Cette intention pédagogique vise aussi à engager tôt la pratique réflexive afin qu'elle soit plus facilement adoptée lors de l'entrée à l'exercice de la profession. Enfin, le compagnonnage cognitif est une approche pédagogique qui suggère un accompagnement propice au développement du RCI. Il pourrait contribuer à une culture de formation continue tout en favorisant l'apport d'une communauté d'apprentissage en sciences infirmières. 


\section{Liste des références}

Alfaro-LeFevre, R. (2013). Critical Thinking, Clinical Reasoning, and Clinical Judgment EBook: A Practical Approach. (5 éd.). St. Louis, MI: Elsevier Health Sciences.

Argyris, C., et Schön, D. (1992/1974). Theory in Practice: Increasing Professionnal Effectiveness. San Francisco, CA: Jossey-Bass.

Audétat, M.-C., Laurin, S., et Sanche, G. (2011). Aborder le raisonnement clinique du point de vue pédagogique. Pédagogie Médicale, 12(4), 223-229. https://doi.org/10.1051/pmed/2011109

Audetat, M. C., Laurin, S., Sanche, G., Beique, C., Fon, N. C., Blais, J. G., et Charlin, B. (2013). Clinical Reasoning Difficulties: A Taxonomy for Clinical Teachers. Medical Teacher, 35(3), e984-989. https://doi.org/10.3109/0142159x.2012.733041

Bédard, D., Frenay, M., Turgeon, J., et Paquay, L. (2000). Les fondements des dispositifs pédagogiques visant à favoriser le transfert de connaissances: les perspectives de l'apprentissage et de l'enseignement contextualisés authentiques. Res Academica, 18(12), 21-46.

Benner, P., Sutphen, M., Leonard, V., et Day, L. (2010). Educating Nurses: A Call for Radical Transformation. San Francisco, CA: Jossey-Bass.

Bisonnette, S., et Richard, M. (2005). Le cognitivisme et ses implications pédagogiques. Dans C. Gauthier et M. Tardif (dir.), La Pédagogie. Théories et pratiques de l'Antiquité à nos jours ( $2^{\mathrm{e}}$ éd., p. 309-332). Montréal, QC: Gaetan Morin Éditeur.

Bordage, G. (1994). Elaborated knowledge: a key to successful diagnostic thinking. Academic Medicine, 69(11), 883-885. http://dx.doi.org/10.1097/00001888-199411000-00004

Boyer, L., Tardif, J., et Lefebvre, H. (2015). From a Medical Problem to a Health Experience: How Nursing Students Think in Clinical Situations. Journal of Nursing Education, 54(11), 625-632. https://doi.org/10.3928/01484834-20151016-03

Brown, J. S., Collins, A., et Duguid, P. (1989). Situated Cognition and the Culture of Learning. Educational researcher, 18(1), 32-42. doi: https://doi.org/10.2307/1176008

Charlin, B., et Fernandez, N. (2016). Préparer et animer une formation par concordance. Dans T. Pelaccia (dir.), Comment (mieux) former et évaluer les étudiants en médecine et en sciences de la santé? (p. 325-340). Bruxelles, Belgique: De Boeck Supérieur.

Charlin, B., Gagnon, R., Sibert, L., et Van der Vleuten, C. (2002). Le test de concordance de script, un instrument d'évaluation du raisonnement clinique. Pédagogie Médicale, 3(3), 135-144. http://dx.doi.org/10.1051/pmed:2002022

Charlin, B., Tardif, J., et Boshuizen, H. P. A. (2000). Scripts and medical diagnostic knowledge: Theory and applications for clinical reasoning instruction and research. Academic Medecine, 75(2), 182-190. http://dx.doi.org/10.1097/00001888-200002000-00020

Charlin, B., et Van der Vleuten, C. (2004). Standardized Assessment in Context of Uncertainty: The Script Concordance Approach. Evaluation and the Health Professions, 26, 304-319. https://doi.org/10.1177/0163278704267043 
Collins, A., Brown, J. S., et Holum, A. (1991). Cognitive apprenticeship: Making thinking visible. American Educator, 15(3), 6-11.

Collins, A., Brown, J. S., et Newman, S. E. (1989). Cognitive Apprenticeship: Teaching the Crafts of Reading, Writing, and Mathematics. Dans L. B. Resnick (dir.), Knowing, Learning and Instruction (p. 453-494). Hillsdale, NJ: Lawrence Erlbaum Associates.

Custers, E. J. (2015). Thirty years of illness scripts: Theoretical origins and practical applications. Medical Teacher, 37(5), 457-462. http://dx.doi.org/10.3109/0142159X.2014.956052

Dennen, V. P., et Burner, K. J. (2008). The cognitive apprenticeship model in educational practice. Handbook of Research on Educational Communications and Technology, 3, 425-439.

Deschênes, M.-F., et Goudreau, J. (2017). Addressing the development of both knowledge and clinical reasoning in nursing through the perspective of script concordance: an integrative literature review. Journal of nursing Education and Practice, 7(12), 28-38. https://doi.org/10.5430/jnep.v7n12p28

Ericsson, K. A. (2008). Deliberate practice and acquisition of expert performance: a general overview. Academic Emergency Medicine, 15(11), 988-994. https://doi.org/10.1111/j.1553-2712.2008.00227.x

Fernandez, N., Foucault, A., Dubé, S., Robert, D., Lafond, C., Vincent, A.-M., ... Charlin, B. (2016). Learning-by-Concordance (LbC): introducing undergraduate students to the complexity and uncertainty of clinical practice. Canadian Medical Education Journal, 7(2), e104-e113. Repéré à https://journalhosting.ucalgary.ca/index.php/cmej/article/view/36690/pdf

Field, D. E. (2004). Moving from novice to expert-the value of learning in clinical practice: a literature review. Nurse Education Today, 24(7), 560-565. https://doi.org/10.1016/j.nedt.2004.07.009

Fonteyn, M. E., et Ritter, B. J. (2008). Clinical reasoning in nursing. Dans J. Higgs, M. A. Jones, $\mathrm{S}$. Loftus et N. Chirstensen (dir.), Clinical reasoning in the health professions ( $3^{\mathrm{e}}$ éd., p. 235-244). Oxford, United Kingdom: Butterworth-Heinemann.

Forneris, S. G., et Peden-McAlpine, C. (2009). Creating context for critical thinking in practice: the role of the preceptor. Journal of Advanced Nursing, 65(8), 1715-1724. http://dx.doi.org/10.1111/j.1365-2648.2009.05031.x

Foucault, A., Dubé, S., Fernandez, N., Gagnon, R., et Charlin, B. (2015). Learning medical professionalism with the online concordance-of-judgment learning tool (CJLT): A pilot study. Medical Teacher, 37(10), 955-960. http://dx.doi.org/10.3109/0142159X.2014.970986

Frenay, M., et Bédard, D. (2004). Des dispositifs de formation universitaire s'inscrivant dans la perspective d'un apprentissage et d'un enseignement contextualisé pour favoriser la construction de connaissances et leur transfert. Dans A. Presseau et M. Frenay (dir.), Le transfert des apprentissages : comprendre pour mieux intervenir (p. 241-268). Québec, QC: Les Presses de 1'Université Laval. 
Frith, C. (2007). Making up the mind: How the brain creates our mental world. Malden, MA: Blackwell Publishing.

Gieselman, J. A., Stark, N., et Farruggia, M. J. (2000). Implications of the situated learning model for teaching and learning nursing research. The Journal of Continuing Education in Nursing, 31(6), 263-268.

Goudreau, J., Boyer, L., et Létourneau, D. (2014). Clinical Nursing Reasoning in Nursing Practice: A Cognitive Learning Model based on a Think Aloud Methodology. Quality Advancement in Nursing Education - Avancées en formation infirmière, 1(1), 1-18. http://dx.doi.org/10.17483/2368-6669.1009

Hoffman, K. A., Aitken, L. M., et Duffield, C. (2009). A comparison of novice and expert nurses' cue collection during clinical decision-making: Verbal protocol analysis. International Journal of Nursing Studies, 46, 1335-1344. https://doi.org/10.1016/j.ijnurstu.2009.04.001

Jouquan, J., Romanus, C., Vierset, V., Jafferelot, M., et Parent, F. (2013). Promouvoir les pédagogies actives comme soutien à la pratique réflexive et à l'apprentissage en profondeur. Dans F. Parent et J. Jouquan (dir.), Penser la formation des professionnels de la santé : une perspective intégrative (p. 245-283). Bruxelles, Belgique : De Boeck supérieur.

Kuiper, R. A. (2013). Integration of innovative clinical reasoning pedagogies into a baccalaureate nursing curriculum. Creative Nursing, 19(3), 128-139. https://doi.org/10.1891/1078-4535.19.3.128

Lasnier, F. (2000). Réussir la formation par compétences. Montréal, QC : Guérin Éditeur.

Lave, J. (1997). The culture of acquisition and the practice of understanding. Dans D. Kirshner et J. A. Whitson (dir.), Situated cognition: Social, Semiotic and Psychological Perspectives (p. 17-35). Mahwah, NJ: Lawrence Erlbaum Associates.

Lave, J., et Wenger, E. (1991). Situated learning: Legitimate peripheral participation. New York, NY: Cambridge University Press.

Lavoie, P., Boyer, L., Pepin, J. I., Goudreau, J., et Fima, O. (2017). Accompagner les infirmières et les étudiantes dans la réflexion sur des situations de soins: Un modèle pour les formateurs en soins infirmiers. Quality Advancement in Nursing Education-Avancées en formation infirmière, 3(1), Article 3, 1-7. https://doi.org/10.17483/2368-6669.1100

Lecourt, D. (2010). Dictionnaire d'histoire et philosophie des sciences (4 éd.). Paris, France : Quadrige/PUF.

Legendre, M.-F. (2004). Cognitivisme et socioconstructivisme. Dans P. Jonnaert et A. M'Batika (dir.), Les réformes curriculaires: regards croisés (p. 13-47). Sainte-Foy, QC : Presses de l'Université du Québec.

Legendre, M.-F. (2005). Lev Vygotsky et le socioconstructivisme en éducation. Dans C. Gauthier et M. Tardif (dir.), La Pédagogie. Théories et pratiques de l'Antiquité à nos jours (2 éd., p. 351-373). Montréal, QC : Gaetan Morin Éditeur.

Levett-Jones, T., Hoffman, K., Dempsey, J., Jeong, S., Noble, D., Norton, C. A., ... et Hickey, N. (2010). The 'five rights' of clinical reasoning: an educational model to enhance 
nursing strudent's ability to identify and manage clinical 'at risk' patients. Nurse Education Today, 30(6), 515-520. http://dx.doi.org/10.1016/j.nedt.2009.10.020

Lubarsky, S., Dory, V., Audétat, M.-C., Custers, E., et Charlin, B. (2015). Using script theory to cultivate illness script formation and clinical reasoning in health professions education. Canadian Medical Education Journal, 6(2), e61-e70. Repéré à https://www.ncbi.nlm.nih.gov/pmc/articles/PMC4795084/

Lyons, K., McLaughlin, J. E., Khanova, J., et Roth, M. T. (2016). Cognitive Apprenticeship in Health Sciences Education: A Qualitative review. Advances in Health Sciences Education, 22(3), 723-739. https://doi.org/10.1007/s10459-016-9707-4

Mandeville, L. (2009). Une expérience d'apprentissage significatif pour l'étudiant. Dans D. Bédard et J.-P. Béchard (dir.), Innover dans l'enseignement supérieur. Paris, France : Presses universitaires de France.

Nendaz, M., Charlin, B., Leblanc, V., et Bordage, G. (2005). Le raisonnement clinique : données issues de la recherche et implications pour l'enseignement. Pédagogie Médicale, 6(4), 235-254. http://dx.doi.org/10.1051/pmed:2005028

Nickle, P. (2006). Cognitive apprenticeship: laying the groundwork for mentoring registered nurses in the intensive care unit. Dynamics, 18(4), 19-27.

O'Brien, V. H., Marks, M. B., et Charlin, B. (2003). La rétroaction (ou rétro-action): un élément de l'intervention pédagogique en milieu clinique. Pédagogie Médicale, 4(3), 184-191. https://doi.org/10.1051/pmed:2003008

Palmeri, T. J. (1997). Exemplar similarity and the development of automaticity. Journal of Experimental Psychology: Learning, Memory, and Cognition, 23(2), 324. https://doi.org/10.1037/0278-7393.23.2.324

Poteaux, N., et Pelaccia, T. (2016). Favoriser le transfert des apprentissages de la salle de cours au milieu de soins. Dans T. Pelaccia (dir.), Comment mieux former et évaluer les étudiants en médecine et en sciences de la santé (p. 169-180). Bruxelles, Belgique: De Boeck Supérieur.

Rieder, A., Poletti, A. H., et Audétat, M.-C. (2015). Clinicien enseignant en cabinet médical: méthodes d'enseignement. Primarycare-Le journal suisse des médecins de premier recours, 15(20), 362-365. https://doi.org/10.4414/pc-f.2015.00846

Sadhuwong, K., Koraneekij, P., et Natakuatoong, O. (2016). Effects of a blended learning model integrating situated multimedia lessons and cognitive apprenticeship method on the clinical reasoning skills of nursing students. Journal of Health Research, 30(6), 421-431.

Sarsfield, E. (2014). Differences Between Novices' and Experts' Solving Ill-Structured Problems. Public Health Nursing, 31(5), 444-453. https://doi.org/10.1111/phn.12100

Schank, R., et Abelson, R. (1977). Script, Plans, Goals and Understanding: An Inquiry into Human Knowledge Structures. Hillsdale, NJ : Lawrence Erlbaum Associates.

Schmidt, H. G., Norman, G. R., et Boshuizen, H. P. A. (1990). A cognitive perspective on medical expertise. Theory and implications. Academic Medecine, 65(10), 611-621. http://dx.doi.org/10.1097/00001888-199010000-00001 
Simmons, B. (2010). Clinical reasoning: concept analysis. Journal of Advanced Nursing, 66(5), 1151-1158. http://dx.doi.org/10.1111/j.1365-2648.2010.05262.x

Simmons, B., Lanuza, D., Fonteyn, M., Hicks, F., et Holm, K. (2003). Clinical reasoning in experienced nurses. Western Journal of Nursing Research, 25(6), 701-724. https://doi.org/10.1177/0193945903253092

Tardif, J. (1992). Pour un enseignement stratégique l'apport de la psychologie cognitive. Montréal, QC : Les Éditions Logiques.

Tardif, J. (1999). Le transfert des apprentissages. Montréal, QC : Les Éditions logiques.

Tardif, J. (2016). Ancrer les apprentissages sur les connaissances antérieures des étudiants. Dans T. Pelaccia (dir.), Comment (mieux) former et évaluer les étudiants en médecine et en sciences de la santé? (p. 149-167). Bruxelles, Belgique: De Boeck Supérieur.

Thompson, C., Aitken, L., Doran, D., et Dowding, D. (2013). An agenda for clinical decision making and judgement in nursing research and education. International Journal of Nursing Studies, 50(12), 1720-1726. https://doi.org/10.1016/j.ijnurstu.2013.05.003

Vanpee, D., Frenay, M., Godin, V., et Bédard, D. (2010). Ce que la perspective de l'apprentissage et de l'enseignement contextualisés authentiques peut apporter pour optimaliser la qualité pédagogique des stages d'externat. Pédagogie Médicale, 10(4), 253-266. https://doi.org/10.1051/pmed/20090330

Vygotsky, L. (1933/2013). Pensée et langage. [Translation of the original text by Françoise Sève ]. (Traduit par F. Sève, $4^{e}$ éd.). Paris, France : La Dispute.

Vygotsky, L. S. (1978). Mind in society: The Development of Higher Psychological Processes. Cambridge, MA : Harvard University Press.

Wenger, E. (1998). Communities of practice: Learning, meaning, and identity. Cambridge, UK: Cambridge University Press.

Woolley, N. N., et Jarvis, Y. (2007). Situated cognition and cognitive apprenticeship: A model for teaching and learning clinical skills in a technologically rich and authentic learning environment. Nurse Education Today, 27(1), 73-79. https://doi.org/10.1016/j.nedt.2006.02.010

Wyrostok, L. H., Hoffart, J., Kelly, I., et Ryba, K. (2014). Situated Cognition as a Learning Framework for International End-of-Life Simulation. Clinical Simulation in Nursing, 10(4), e217-e222. http://dx.doi.org/10.1016/j.ecns.2013.11.005 